\title{
Quantum Renewal Equation for the first detection time of a quantum walk
}

\author{
H. Friedman, ${ }^{1}$ D. A. Kessler, ${ }^{1}$ and E. Barkai ${ }^{1}$ \\ ${ }^{1}$ Department of Physics, Institute of Nanotechnology and Advanced Materials, Bar Ilan University, Ramat-Gan 52900, Israel
}

\begin{abstract}
We investigate the statistics of the first detected passage time of a quantum walk. The postulates of quantum theory, in particular the collapse of the wave function upon measurement, reveal an intimate connection between the wave function of a process free of measurements, i.e. the solution of the Schrödinger equation, and the statistics of first detection events on a site. For stroboscopic measurements a quantum renewal equation yields basic properties of quantum walks. For example, for a tight binding model on a ring we discover critical sampling times, diverging quantities such as the mean time for first detection, and an optimal detection rate. For a quantum walk on an infinite line the probability of first detection decays like (time) $)^{-3}$ with a superimposed oscillation, critical behavior for a specific choice of sampling time, and vanishing amplitude when the sampling time approaches zero due to the quantum Zeno effect.
\end{abstract}

A century ago Schrödinger [1] discovered a fundamental connection between occupation probabilities and first passage time distributions [2, 3], a relationship used to derive some of the most basic properties of classical random walks. Ever since, the problem of first passage time statistics has attracted tremendous interest, as it is applicable to many fields of science [4, 5]. In a nutshell the main idea [1 is simple: the path of a Markovian random walk on a graph, starting at $x$ and reaching 0 at time $t$, can be decomposed into a path that arrived at 0 for the first time at time $t^{\prime}$, and then an independent segment which starts at 0 and returns back in the time interval $t-t^{\prime}$. The consequence is a well known renewal formula, the first equation in Redner's monograph [3, relating occupation probabilities and first passage time statistics. More recently, quantum walks have attracted much interest both theoretically $[6]$ and experimentally [9 11$]$. These exhibit interference patterns and ballistic scaling and in that sense exhibit behaviors drastically different from the classical random walk. Particularly controversial has been the question of the first passage time for quantum dynamics. The latter is ill defined, so we consider the first detected passage time to a site (see details below) 12 19. Our main result is the quantum analogue of Schrödinger's classical renewal equation. With this equation, which deals with amplitudes, rather than probabilities, we calculate some basic properties of quantum walks, for example the first detected passage time statistics on a line. Our approach is based on a projective measurement scheme recently introduced by Dhar et al. [18, 19. We note that the quest for the quantum renewal equation, the question of first passage time in quantum mechanics, or more generally the fluctuations of time observables, has a long hotly debated history. Here we use the textbook postulates of quantum measurements, in particular the projective postulate [20, to address the first detection problem of quantum dynamics, the main restriction being that the Hamiltonian is time independent, the latter corresponding to the Markov assumption used by Schrödinger in the classical domain.

Model and the measurement process. We consider a single quantum particle on a graph, for example a lattice or a discretized ring, whose state wave function is $|\psi\rangle$. Under stroboscopic observations at times $\tau, 2 \tau, \cdots$. an observer performs measurements at a spatial position which we may call $x=0$ which is represented as the vector $|0\rangle$ (see schematic diagram in Fig. 1). Such stroboscopic measurements are useful as they capture quantum periodicities as shown below. A measurement provides two possible outcomes: either the particle is detected at $x=0$ or it is not. The experiment provides the string: no, no, no, $\cdots$ and finally at the $n$-th attempt a yes so that $n \tau$ is the first detected passage time, whose statistics are investigated. For that we must define the measurement process precisely [18, 19].

Just prior to the first measurement the wave function is $\left|\psi\left(\tau^{-}\right)\right\rangle=U(\tau)|\psi(0)\rangle$ and $U(\tau)=\exp (-i H \tau)$ is the unitary evolution operator, $H$ the time independent Hamiltonian, $|\psi(0)\rangle$ is the wave function at the initial time $t=0$ and $\hbar=1$. For example, we will later investigate the tight-binding model

$$
H=-\gamma \sum_{x=-\infty}^{\infty}(|x\rangle\langle x+1|+| x+1\rangle\langle x|) .
$$

This describes a quantum particle jumping between nearest neighbours on an infinite one dimensional lattice so $x$ is an integer and in that sense the model describes a quantum random walk 8 . We stress that our main results are not limited to a specific Hamiltonian.

The probability of finding the particle in state $|0\rangle$, at the first measurement, is $P_{1}=\left|\left\langle 0 \mid \psi\left(\tau^{-}\right)\right\rangle\right|^{2}$. If the outcome of the first measurement is positive we get $n=1$. On the other hand, if the particle is not detected, with probability $1-P_{1}$, Von Neumann's postulate of collapse states that the null measurement alters the wave function in such a way that the probability of detecting the particle at the detection point $x=0$ at time $\tau^{+} \equiv \tau+0^{+}$ is zero. Afterwards, the evolution of the quantum state will resume until the next measurement time $2 \tau$ via the transformation $U(\tau)$. In this sense we are considering projective measurements whose duration is very short, 


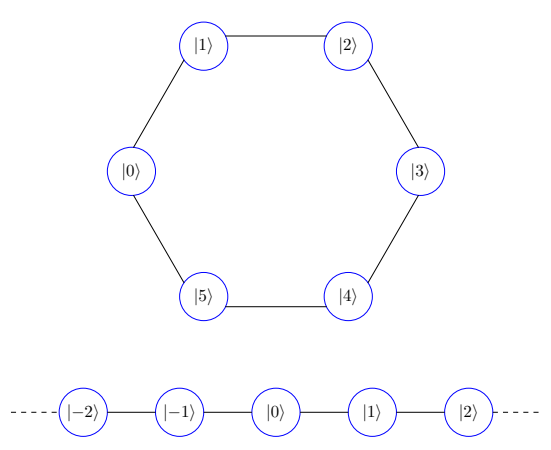

FIG. 1. Schematic models of a benzene-like ring, representing a closed geometry, and an infinite one dimensional lattice. Measurements on site $x=0$ are made stroboscopically at time $\tau, 2 \tau, \ldots$ to capture periodicities in the underlying dynamics.

while between the measurements the evolution is according to the Schrödinger equation.

Since the outcome of a null measurement is zero amplitude for finding the particle at $x=0$ at time $\tau^{+}$we have

$$
\left|\psi\left(\tau^{+}\right)\right\rangle=N(\mathbb{1}-|0\rangle\langle 0|)\left|\psi\left(\tau^{-}\right)\right\rangle,
$$

where $\mathbb{1}$ is the identity operator, and $N$ is determined from the normalization condition. Since just prior to the measurement the probability of finding the particle at $x \neq 0$ is $1-P_{1}$, we get 20 .

$$
\left|\psi\left(\tau^{+}\right)\right\rangle=\frac{\mathbb{1}-\hat{D}}{\sqrt{1-P_{1}}} U(\tau)|\psi(0)\rangle .
$$

where $\hat{D}=|0\rangle\langle 0|$ is the measurement's projection operator. The probability of detecting the particle at the second measurement, conditioned that the quantum walker was not found in the first attempt, is $P_{2}=$ $\left|\left\langle 0|U(\tau)| \psi\left(\tau^{+}\right)\right\rangle\right|^{2}$. This procedure is continued to find the probability of first detection in the $n$-th measurement, conditioned that prior measurements did not detect the particle [18, 19,

$$
P_{n}=\frac{\left|\left\langle 0\left|[U(\tau)(1-\hat{D})]^{n-1} U(\tau)\right| \psi(0)\right\rangle\right|^{2}}{\left(1-P_{1}\right) \ldots\left(1-P_{n-1}\right)} .
$$

In the numerator (respectively, the denominator) the operator $1-\hat{D}$ (the probabilities of non-detection $1-P_{j}$ ) is found $n-1$ times corresponding to the $n-1$ prior measurements.

First detection probability $F_{n}$. The main focus of this work is on the probability of first detection in the $n$-th attempt, denoted $F_{n}$. This detection consists of a set of $n-1$ null measurements, each weighed by the conditional probability $1-P_{j}$, followed by a positive measurement at attempt $n$, giving

$$
F_{n}=\left(1-P_{1}\right)\left(1-P_{2}\right) \ldots\left(1-P_{n-1}\right) P_{n} .
$$

Using Eq. (4), $F_{n}=\left|\phi_{n}\right|^{2}$ where

$$
\phi_{n}=\left\langle 0\left|U(\tau)[(1-\hat{D}) U(\tau)]^{n-1}\right| \psi(0)\right\rangle
$$

is the first detection amplitude.

Solution using generating functions. Eq. (6) gives $\phi_{1}=\langle 0|U(\tau)| \psi(0)\rangle, \phi_{2}=\langle 0|U(2 \tau)| \psi(0)\rangle-\phi_{1}\langle 0|U(\tau)| 0\rangle$ and by induction we find

$$
\phi_{n}=\langle 0|U(n \tau)| \psi(0)\rangle-\sum_{j=1}^{n-1} \phi_{j}\langle 0|U[(n-j) \tau]| 0\rangle .
$$

This iteration rule yields the amplitude $\phi_{n}$ in terms of a propagation free of measurement, i.e. $\langle 0|U(n \tau)| \psi(0)\rangle$ is the amplitude for being at the origin at time $n \tau$ in the absence of measurements, from which we subtract $n-1$ terms related to the previous null measurements of the particle. In practice, it is more convenient to work in terms of the generating function 21] also called the $Z$ transform $\hat{\phi}(z) \equiv \sum_{n=1}^{\infty} z^{n} \phi_{n}$. Multiplying Eq. (7) by $z^{n}$ and summing over $n$ using the convolution theorem we get

$$
\hat{\phi}(z)=\frac{\langle 0|\hat{U}(z)| \psi(0)\rangle}{1+\langle 0|\hat{U}(z)| 0\rangle},
$$

where $\hat{U}(z) \equiv \sum_{n=1}^{\infty} z^{n} U(n \tau)$. From the generating function we obtain useful information on the process using inversion formulae provided in the supplementary material (SM).

Quantum renewal equation. For a particle free of any measurement, the amplitude of being at the origin at time $t$ is $\left\langle 0 \mid \psi_{f}(t)\right\rangle$ and $\left|\psi_{f}(t)\right\rangle=\exp (-i H t)\left|\psi_{f}(0)\right\rangle$. Here $\left|\psi_{f}(t)\right\rangle$ is the solution of the Schrödinger equation $i \partial_{t}\left|\psi_{f}\right\rangle=H\left|\psi_{f}\right\rangle$, with the same initial conditions as for the first detection problem under investigation $\left|\psi_{f}(0)\right\rangle=|\psi(0)\rangle$. Let us consider the initial condition where the particle is initially localized at $x=0$ and so $|\psi(0)\rangle=|0\rangle$. Using $\langle 0 \mid 0\rangle=1$, Eq. (8) is rewritten

$$
\hat{\phi}(z)=1-\frac{1}{\left\langle 0\left|\frac{1}{1-z e^{-i H \tau}}\right| 0\right\rangle} .
$$

We define the measurement-free generating function

$$
\left\langle 0 \mid \psi_{f}(z)\right\rangle_{0} \equiv \sum_{n=0}^{\infty} z^{n}\left\langle 0 \mid \psi_{f}(n \tau)\right\rangle,
$$

and clearly $\left\langle 0 \mid \psi_{f}(z)\right\rangle_{0}=\sum_{n=0}^{\infty}\left\langle 0\left|z^{n} \exp (-i H \tau n)\right| 0\right\rangle$, the subscript zero denoting the initial condition. Summing the geometric series, we get the appealing result

$$
\hat{\phi}(z)=1-\frac{1}{\left\langle 0 \mid \psi_{f}(z)\right\rangle_{0}} .
$$

Thus, the generating function of the first detection time amplitude $\hat{\phi}(z)$ is determined from the $Z$ transform of the wave function at the point of detection $x=0$. 


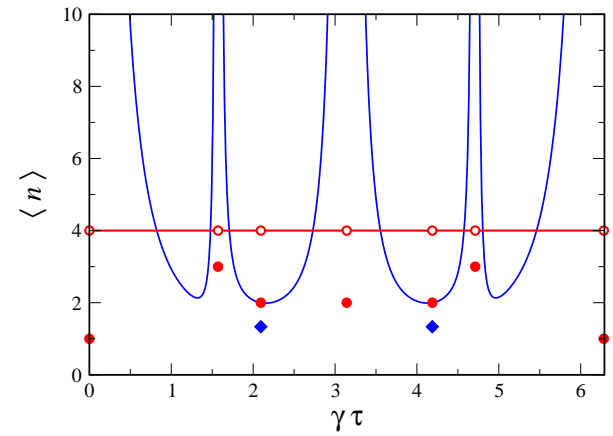

FIG. 2. The average first successful detection $\langle n\rangle$ versus $\gamma \tau$ for a benzene-like ring where the starting point is on $x=3$ (blue) or $x=0$ (red) and the detection is on $x=0$.

Similarly, for an initial condition initially localized at a site $x \neq 0$, so that $|\psi(0)\rangle=|x\rangle$, for detection at site 0 we find that

$$
\hat{\phi}(z)=\frac{\left\langle 0 \mid \psi_{f}(z)\right\rangle_{x}}{\left\langle 0 \mid \psi_{f}(z)\right\rangle_{0}}
$$

where $\left|\psi_{f}(z)\right\rangle_{x}$ is the $Z$ transform of the wave function free of measurements initially localized on site $x,\left|\psi_{f}(z)\right\rangle_{x}=\sum_{n=0}^{\infty} z^{n}\left|\psi_{f}(n \tau)\right\rangle_{x}$ with $\left|\psi_{f}(n \tau)\right\rangle_{x}=$ $\exp (-i H n \tau)|x\rangle$. Eqs. 111 12 are the quantum counterparts of the classical renewal equation Eq. 1.2.3 (or Eq. I.18) in 2, 3] respectively. The latter deals with the correspondence between occupation and first passage time probabilities, while we have found the connection between the amplitudes $\phi_{n}$ and the wave function $\left|\psi_{f}\right\rangle$. In that sense we have reduced the problem of first detection time to the computation of the $Z$ transform of the solution of the Schrödinger equation.

Rings. We first consider a tight-binding ring with $L$ sites, namely the Hamiltonian Eq. (1) with periodic boundary conditions. The solution of the Schrödinger equation $\left|\psi_{f}\right\rangle$ is computed with standard methods. To find $\phi_{n}$ we use the inverse $Z$ transform (see SM). For an $x=0$ initial condition, i.e. $|\psi(0)\rangle=|0\rangle$, where $x=0$ is also the location of the detector, we find the following three results: (i) The particle is detected with probability 1 and in this sense the quantum walk is recurrent. (ii) Besides isolated sampling times $\tau$ listed below, the average number of detection attempts is

$$
\langle n\rangle= \begin{cases}\frac{L+2}{2} & L \text { is even } \\ \frac{L+1}{2} & L \text { is odd }\end{cases}
$$

This result is remarkable since it is independent of the sampling time $\tau$. (iii) Exceptional sampling times $\tau$ are given by the rule

$$
\Delta E \tau=2 \pi n,
$$

where $n$ is a non-negative integer, and $\Delta E=E_{i}-E_{j}>0$ is the energy difference between pairs of eigenenergies of the underlying Hamiltonian. These exceptional points exhibit non-analytical behaviors, diverging moments of $n$ and critical slowing down, as we now discuss.

For example, for a benzene-like ring of size $L=6$, schematically shown in the upper part of Fig. 1. Eq. (13) gives $\langle n\rangle=4$. The energy levels are $\pm \gamma$ and $\pm 2 \gamma$, the former are doubly degenerate. Using Eq. (11) we find $\langle n\rangle=1,3,2,2, \cdots$ for the exceptional points given by Eq. (14) $\gamma \tau=0, \pi / 2,2 \pi / 3, \pi \cdots$ which is continued periodically, see Fig. 2. Physically, the condition Eq. (14) implies a partial revival of the wave packet free of measurement, namely two modes of the system are behaving identically when strobed at period $\tau$. When $\tau=0$ we get the expected result, the particle is detected immediately and then $\langle n\rangle=1$, which is also found when $\gamma \tau=2 \pi$, namely at a full revival period.

Exceptional sampling times manifest themselves in different ways depending on the observable and the initial condition. For example, consider again the average $\langle n\rangle$ for the benzene-like ring but now with the initial condition that the particle is localized at the site $x=3$ (see Fig. 11. Eq. (12) gives, except for the exceptional $\tau \mathrm{s}$,

$\langle n\rangle=\frac{27+23 \cos (\gamma \tau)+24 \cos (2 \gamma \tau)+9 \cos (3 \gamma \tau)-2 \cos (4 \gamma \tau)}{9 \sin ^{2}(2 \gamma \tau)}$.

The result, presented in Fig. 2, shows that $\langle n\rangle$ diverges when $\sin (2 \gamma \tau) \rightarrow 0$. When $\tau \rightarrow 0$ the measurements become very frequent and then the probability of measuring the particle approaches zero and so $\langle n\rangle$ diverges, which is the manifestation of the quantum Zeno effect 22. A similar blowup of $\langle n\rangle$ is observed when $\gamma \tau \rightarrow 2 \pi$, since the wave packet fully revives on its initial position $x=3$ and so the measurements do not detect the particle. Similarly $\langle n\rangle$ diverges also for $\gamma \tau$ approaching $\pi / 2, \pi, 3 \pi / 2$ due to partial revivals. A far more subtle effect takes place on the special sampling times $\gamma \tau=2 \pi / 3,4 \pi / 3$. There $\langle n\rangle$ exhibits a discontinuity: on these exceptional points $\langle n\rangle=4 / 3$ while in their vicinity we find from Eq. (15) $\langle n\rangle \simeq 2$. Thus the effects of exceptional points on observables are non-trivial. We have found several other peculiar behaviors for rings [23, but now we turn to the case of an unbounded quantum walk, since the corresponding classical problem is fundamental in stochastic theories, e.g., it gives the random walk exponents through the long tailed first passage PDF [1]. Note that Bach, et al. 13] treated the detection problem for a discrete time Hadamard quantum walk, leading to behaviors different from what we find here.

First detection time for an unbounded quantum walk described by the tight binding Hamiltonian Eq. (1) is now investigated. For a particle starting at the origin, 
we use $\left\langle 0 \mid \psi_{f}(t)\right\rangle=J_{0}(2 \gamma t)$ [8, 17] and Eq. 11]

$$
\hat{\phi}(z)=1-\frac{1}{\left\langle 0 \mid \psi_{f}(z)\right\rangle_{0}}=1-\frac{1}{\sum_{n=0}^{\infty} z^{n} J_{0}(2 \gamma \tau n)},
$$

where $J_{0}(x)$ is the Bessel function of the first kind. Employing $J_{0}(2 \gamma \tau n) \sim \cos (2 \gamma \tau n-\pi / 4) / \sqrt{\pi \gamma \tau n}$ we obtain the large $n$ limit of $\phi_{n}$. From this asymptotic property of the Bessel function it becomes clear that the generating function $\left\langle 0 \mid \psi_{f}(z)\right\rangle_{0}$ does not converge when $z=r \exp (i \theta)$ with $\theta= \pm 2 \gamma \tau$ and $r \geq 1$. Thus, as shown in the SM, when we invert $\hat{\phi}(z)$ to find $\phi_{n}$, we find two branch cuts in the complex $(r, \theta)$ plane. These branch cuts merge when $2 \gamma \tau$ is an integer multiple of $\pi$, a mathematical observation which is behind the critical behavior we find below.

In the SM we derive another one of our main results: the probability of measuring the quantum walker returning to its origin for the first time after $n$ attempts

$$
F_{n} \sim \frac{4 \gamma \tau}{\pi n^{3}} \cos ^{2}\left(2 \gamma \tau n+\frac{\pi}{4}\right) .
$$

This formula, which is valid for large $n$, is the quantum version of the first passage time problem of a classical one dimensional non-biased walker which exhibits the well known power law and monotonic tail $F_{\text {classical }} \propto n^{-3 / 2}$ [1, 3. The role of sampling time in the quantum problem is crucial. When $\tau \rightarrow 0$ the prefactor of the $n^{-3}$ power law in Eq. (17) vanishes, a manifestation of the quantum Zeno effect (a similar effect is found for all initial conditions). Furthermore, the formula predicts that when $\gamma \tau / \pi$ is rational the probability $F_{n}$ multiplied by $n^{3}$ is periodic. In contrast if $\gamma \tau / \pi$ is not rational the asymptotic behavior appears irregular (see Fig. 3). In the limit $2 \gamma \tau \rightarrow \pi$ Eq. (17) gives $F_{n} \sim n^{-3}$ which is a pure power law. However the sampling time $2 \gamma \tau=\pi$ is exceptional and for this case a detailed calculation reveals $F_{n} \sim n^{-3} / 4$ so a factor of 4 mismatch is found (see SM). In this sense exceptional points are found also for an infinite system. This in turn implies a critical slowing down when $2 \gamma \tau \simeq \pi$ (see SM), a behavior that cannot be anticipated without a detailed calculation. Physically, the energy band width of a ring of size $L \rightarrow \infty$ is $\Delta E=4 \gamma$ and inserting that in Eq. 14 with $n=1$ we get the exceptional sampling time of the infinite system.

Summary. We have derived the long sought after quantum renewal equation, obtaining the first detection probability of an unbounded quantum random walk in one dimension, and finding unusual non-analytical behavior even for a small benzene-like ring. Our results are thus the quantum version of Schrödinger's pioneering work on the classical first passage time problem from a century ago [1. The applications of our main formulas are vast, since they are not limited to a specific Hamiltonian. We note that stroboscopic sampling is very useful in quantum systems, since this reveals revivals, critical

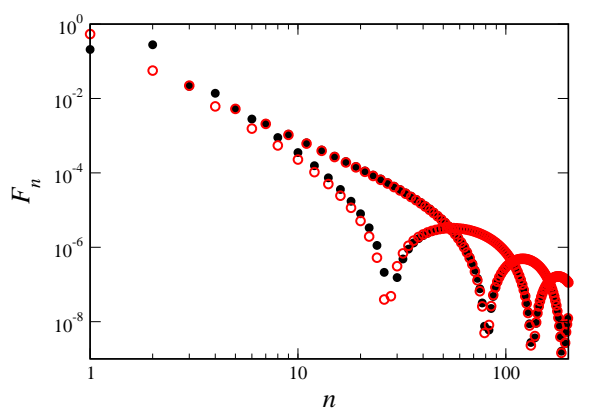

FIG. 3. $\quad F_{n}$ versus $n$ for $\gamma \tau / \pi=0.8 / \pi$, for a quantum walk on a one dimensional lattice. The asymptotic result Eq. 17) (red open circles) matches the exact solution already for moderate value of $n$. The exact solution (full dots) is obtained using a Taylor series of $\hat{\phi}(z)$ Eq. 16 the $n$-th term yields $\phi_{n}$ (see details in SM).

points, and periodicities, though in principle the method used in this work could be extended to other measurement protocols.

Acknowledgement We thank the Israel Science Foundation for funding.

[1] E. Schrödinger, Z. Phys. 16, 289 (1915).

[2] E. Montroll and G. H. Weiss, J. Math. Phys. 6, 167 (1965).

[3] S.Redner, A Guide to First-Passage Processes (Cambridge University Press, Cambridge 2007).

[4] R. Metzler, G. Oshanin, and S. Redner editors, First passage phenomena and their applications, (World Scientific, Singapore 2014).

[5] O. Benichou, . Guérin, and R. Voituriez, J. of Phys. A: Math. and Theoretical 18(16), 1630001 (1-43) (2015).

[6] Y. Aharonov, L. Davidovich, and N. Zagury, Phys. Rev. A. 48, 1687 (1993).

[7] A. Ambainis, E. Bach, A. Nayak, A. Vishwanath, and J. Watrous, in Proceedings of the 33rd Annual ACM Symposium on Theory of Computing (ACM Press, New York, 2001), pp. 3749.

[8] O. Mülken and A. Blumen, Phys. Rep. 50237 (2011).

[9] H. B. Peretrsz, Y. Lahini, F. Pozzi, M. Sorel, R. Morandotti, and Y. Silberberg, Phys. Rev. Lett. 100, 170506 (2008).

[10] M. Karski, et al. Science 235, 5937 (2009).

[11] P. Xue, R. Zhang, H. Qin, X. Zhan, Z. H. Bian, J. Li, and B. C. Sanders, Phys. Rev. Lett. 114, 140502 (2015).

[12] O. Lumpkin, Phys. Rev. A 512758 (1995).

[13] E. Bach, S. Coppersmith, M. P. Goldschen, R. Joynt, and J. Watrous, J. of Computer and System Sciences 69, 562 (2004).

[14] M. Stefanak, I. Jex, and T. Kiss, Phys. Rev. Lett. 100, 020501 (2008).

[15] S. Goswami, P. Sen, and A. Das, Phys. Rev. E. 81, 
$021121(2010)$.

[16] M. Montero, Phys. Rev. A. 88, 012333 (2013).

[17] P. L. Krapivsky, J. M. Luck, and K. Mallick, J. Stat. Phys. 154, 1430 (2014).

[18] S. Dhar, S. Dasgupta, A. Dhar, and D. Sen, Phys. Rev. A. 91, 062115 (2015).

[19] S. Dhar, S. Dasgupta, and A. Dhar, J. Phys. A 48, 115304 (2015).

[20] C. Cohen-Tannoudji, B. Diu and F. Laloë, Quantum Mechanics (John Wiley and Sons, New York 1977).

[21] F. L. H. Brown, Acc. Chem. Phys. 39, 363 (2006).

[22] B. Misra, and E. C. G. Sudarshan, J. of Mathematical Physics 18, 758 (1977).

[23] H. Friedman, D. A. Kessler, and E. Barkai, (to be published). 


\section{SUPPLEMENTARY MATERIAL}

\section{Generating functions: useful formulas.}

The amplitudes $\phi_{n}$ are given by the inversion formula

$$
\phi_{n}=\left.\frac{1}{n !} \frac{d^{n}}{d z^{n}} \hat{\phi}(z)\right|_{z=0}
$$

or

$$
\phi_{n}=\frac{1}{2 \pi i} \oint_{C} \hat{\phi}(z) z^{-n-1} \mathrm{~d} z
$$

where $C$ is a counter-clockwise path that contains the origin and is entirely within the radius of convergence of $\hat{\phi}(z)$. These well-known equations follow from the definition of the generating function. To find $\phi_{n}$ for a finite $n$, once $\hat{\phi}(z)$ is known, one may use Mathematica to expand $\hat{\phi}(z)=\sum_{n=1}^{\infty} \phi_{n} z^{n}$ evaluating the first $n$ terms. For the examples we analysed in the text this method is both practical and fast for finite though large $n$ (e.g. Fig. 3 in the text).

The eventual probability of being measured is related to the generating function $\hat{\phi}(z)$ by

$$
\begin{gathered}
1-S_{\infty}=\sum_{n=1}^{\infty} F_{n}=\sum_{n=1}^{\infty}\left|\phi_{n}\right|^{2}= \\
\frac{1}{2 \pi} \int_{0}^{2 \pi} \sum_{k=1}^{\infty} \phi_{k} e^{i \theta k} \sum_{l=1}^{\infty} \phi_{l}^{*} e^{-i \theta l} \mathrm{~d} \theta=\frac{1}{2 \pi} \int_{0}^{2 \pi}\left|\hat{\phi}\left(e^{i \theta}\right)\right|^{2} \mathrm{~d} \theta .
\end{gathered}
$$

Similarly,

$$
\langle n\rangle=\sum_{n=1}^{\infty} n F_{n}=\frac{1}{2 \pi} \int_{0}^{2 \pi}\left[\hat{\phi}\left(e^{i \theta}\right)\right]^{*}\left(-i \frac{\partial}{\partial \theta}\right) \hat{\phi}\left(e^{i \theta}\right) \mathrm{d} \theta .
$$

The latter is the average of $n$ only when the particle is detected with probability one, namely when $S_{\infty}=0$. Thus with the knowledge of the generating function we may compute the statistical properties of the first detection problem.

\section{Benzene-like ring}

As we showed in the text, to find $\hat{\phi}(z)$ one must first find $\left|\psi_{f}\right\rangle$, and as well known, this time dependent solution of the Schrödinger equation is governed by the energy spectrum $\left\{E_{k}\right\}$ and eigenstates of the Hamiltonian, together with the initial condition. For a benzene-like ring, with initial condition on $x=0$ we find

$$
\hat{\phi}(z)=\frac{\frac{1}{6} \sum_{k=0}^{5} \frac{1}{z^{-1} \exp \left(i E_{k} \tau\right)-1}}{1+\frac{1}{6} \sum_{k=0}^{5} \frac{1}{z^{-1} \exp \left(i E_{k} \tau\right)-1}} .
$$

The nondegenerate energy levels $\left\{E_{k}\right\}(k=0, \ldots, 5)$ are $-2 \gamma$ and $2 \gamma$ while $-\gamma$ and $\gamma$ are doubly degenerate. We use Eqs. 19, 20, 21) to derive results presented in the text, and similarly for other initial conditions. The integrals can be evaluated analytically (to be published) but also numerical integration provides sufficiently accurate results. We note that the structure of Eq. 222 remains for a ring of any size $L$, namely the energy levels of the system determine the generating function completely, for the case when we start at $x=0$.

Table 1 summarizes the behavior of $S_{\infty}$ for the benzene-like ring. For the initial conditions $x=0$ and $x=3$ we find the classical behavior $S_{\infty}=0$ for nearly all sampling rates. However, on what we call exceptional sampling times $\gamma \tau=0, \pi / 2,2 \pi / 3$ etc we find non-classical behaviour for the initial condition $x=3$. Another feature presented in the table are half dark states, namely states where the particle is found with probability $1 / 2$ for nearly all sampling points. 


\begin{tabular}{c|c|c|c|c|c|c|c|c}
$x$ & $0<\gamma \tau<2 \pi^{*}$ & $\gamma \tau=0$ & $\frac{1}{2} \pi$ & $\frac{2}{3} \pi$ & $\pi$ & $\frac{4}{3} \pi$ & $\frac{3}{2} \pi$ & $2 \pi$ \\
\hline 0 & 0 & 0 & 0 & 0 & 0 & 0 & 0 & 0 \\
1 & $1 / 2$ & 1 & $5 / 6$ & 1 & 1 & 1 & $5 / 6$ & 1 \\
2 & $1 / 2$ & 1 & $1 / 2$ & 1 & $1 / 2$ & 1 & $1 / 2$ & 1 \\
3 & 0 & 1 & $1 / 3$ & 0 & 1 & 0 & $1 / 3$ & 1
\end{tabular}

TABLE I. The survival probability $S_{\infty}$ for a quantum walker on a benzene-type ring for different localized starting points $|\psi(0)\rangle=|x\rangle$. Measurements are at $x=0$ and hence initial conditions on sites 1 and 2 are equivalent to initial conditions on 5 and 4 respectively. $0<\gamma \tau<2 \pi^{*}$ implies all values of $\gamma \tau$ in the interval, besides the exceptional points listed on the top row.

\section{First detection for unbounded particle.}

Here we analyse the large $n$ behavior of $\phi_{n}$ for an unbounded tight binding quantum walk, where initially the walker is localized at the origin. The analysis is performed via integration in the complex plane using Eqs. (16, 19). We define

$$
I(z)=\sum_{\tilde{n}=1}^{\infty} z^{\tilde{n}} J_{0}(2 \gamma \tilde{n} \tau)
$$

and hence the generating function is

$$
\hat{\phi}(z)=\frac{I(z)}{1+I(z)}
$$

As we shall see, the large $n$ behavior of $\hat{\phi}_{n}$ is determined by the singularity structure of $I(z)$, which in turn is controlled by the large $\tilde{n}$ terms in the sum Eq. 23 . In the large $\tilde{n}$ limit the asymptotic behavior of the Bessel function is

$$
J_{0}(2 \gamma \tau \tilde{n}) \sim \frac{\cos (2 \gamma \tau \tilde{n}-\pi / 4)}{\sqrt{\pi \gamma \tau \tilde{n}}}
$$

Thus, we define

$$
I_{\gamma \tau}(z)=\sum_{\tilde{n}=1}^{\infty} z^{\tilde{n}} \frac{\cos (2 \gamma \tau \tilde{n}-\pi / 4)}{\sqrt{\pi \gamma \tau \tilde{n}}}
$$

Then the large $n$ limit of $\phi_{n}$ is given by the inversion of

$$
\hat{\phi}(z) \sim \frac{I_{\gamma \tau}(z)}{1+I_{\gamma \tau}(z)} .
$$

Infinite system $\gamma \tau=\pi / 2$

Considering the case $\gamma \tau=\pi / 2$, we find $I_{\pi / 2}(z)=\pi^{-1} \mathrm{Li}_{1 / 2}(-z)$, where the polylog $\operatorname{Li}_{1 / 2}(z)$ is defined by

$$
\operatorname{Li}_{1 / 2}(z)=\sum_{k=1}^{\infty} \frac{z^{k}}{\sqrt{k}} .
$$

We first investigate the branch cut of this function in the complex plane.

\section{On the Polylog}

We want to understand the behavior of the polylog function near the singularity at $z=1$. The simplest approach is simply to ask Mathematica.

$$
\operatorname{Li}_{1 / 2}(z) \approx \sqrt{\frac{\pi}{1-z}}+\zeta(1 / 2)+\ldots
$$


where $\zeta$ is the Riemann zeta function. Now, for $z$ complex, $z=1+y \pm i \epsilon$, we get

$$
\operatorname{Li}_{1 / 2}(z) \approx \sqrt{\frac{\pi}{-y \mp i \epsilon}}=\sqrt{\frac{\pi}{y e^{\mp i \pi}}}= \pm i \sqrt{\frac{\pi}{y}}
$$

showing the branch cut discontinuity for $z>1$. A simple way to understand this behavior is to note that the singularity structure is identical to that of

$$
f(z) \equiv \int_{0}^{\infty} \frac{d t}{\sqrt{t}} e^{t \ln z}=\Gamma(1 / 2)(-\ln z)^{-1 / 2} .
$$

Thus for $z<1$, this diverges as

$$
f(z) \approx \sqrt{\frac{\pi}{1-z}}
$$

exactly as above. The behavior for $z=1+y \pm i \epsilon$ follows similarly.

Another approach is to calculate the discontinuity across the branch cut. We start from the integral representation of the polylog:

$$
\begin{aligned}
\operatorname{Li}_{1 / 2}(z) & =\frac{1}{\Gamma(1 / 2)} \sum_{k=1}^{\infty} \int_{0}^{\infty} \frac{d t}{\sqrt{t}} e^{-k t} z^{k} \\
& =\frac{1}{\sqrt{\pi}} \int_{0}^{\infty} \frac{d t}{\sqrt{t}} \frac{1}{e^{t / z-1}} .
\end{aligned}
$$

This gives the discontinuity across the branch cut Disc $\left[\operatorname{Li}_{1 / 2}(z)\right]=\operatorname{Li}_{1 / 2}(1+y+i \epsilon)-\operatorname{Li}_{1 / 2}(1+y-i \epsilon)$ where $\epsilon \rightarrow 0^{+}$

$$
\begin{aligned}
\operatorname{Disc}\left[\operatorname{Li}_{1 / 2}(z)\right] & =\lim _{\epsilon \rightarrow 0^{+}} \frac{1}{\sqrt{\pi}} \int_{0}^{\infty} \frac{d t}{\sqrt{t}}\left[\frac{1}{\frac{e^{t}}{1+y}\left(1-i \frac{\epsilon}{1+y}\right)-1}-\frac{1}{\frac{e^{t}}{1+y}\left(1+i \frac{\epsilon}{1+y}\right)-1}\right] \\
& =\lim _{\tilde{\epsilon} \rightarrow 0^{+}} \frac{1}{\sqrt{\pi}} \int_{0}^{\infty} \frac{d t}{\sqrt{t}} \frac{2 i \tilde{\epsilon}}{f^{2}+\tilde{\epsilon}^{2}} \\
& =2 i \sqrt{\pi} \int_{0}^{\infty} \frac{d t}{\sqrt{t}} \delta(f),
\end{aligned}
$$

where we have defined $\tilde{\epsilon} \equiv \epsilon e^{t} /(1+y)^{2}$ and $f \equiv e^{t} /(1+y)-1$. For small positive $y$, we can find the zero, $t_{*}$ of $f$ :

$$
f(t) \approx \frac{1+t}{1+y}-1 \Rightarrow t_{*} \approx y .
$$

Thus, doing the integral over $t$ we get

$$
\operatorname{Disc}\left[\operatorname{Li}_{1 / 2}(z)\right] \approx 2 i \sqrt{\frac{\pi}{y}}
$$

which is consistent with what we have gotten previously.

$$
\text { Undoing the } z \text { Transform for } \gamma \tau=\pi / 2
$$

We want to calculate $\phi_{n}$, using the inverse $z$ transform of

$$
\hat{\phi}(z)=\frac{\operatorname{Li}_{1 / 2}(-z)}{\pi+\operatorname{Li}_{1 / 2}(-z)} .
$$

One can show that there are no poles inside the unit circle, hence the dominant contribution arises from the branch cut of the polylog, namely $\hat{\phi}(z)$ has a branch cut along the negative $z$-axis. Now, as mentioned

$$
\phi_{n}=\oint \frac{d z}{2 \pi i z^{n+1}} \hat{\phi}(z),
$$


where the contour encloses the origin and does not cross the branch cut. We can deform the contour so that it goes counter-clockwise along the circle at infinity from $\theta=-\pi-i \epsilon$ to $\theta=\pi+i \epsilon$, runs along the branch cut from $z=-\infty+i \epsilon$, goes around the point at $z=-1$ in an infinitesimal circle and returns along the bottom of the branch cut back out to infinity. The contribution from the circle at infinity vanishes and so we are left with the integral along the branch cut, so that

$$
\phi_{n}=\int_{-\infty}^{-1} \frac{d z}{2 \pi i z^{n+1}} \operatorname{Disc}[\hat{\phi}(z)]
$$

where as above the discontinuity is taken from above to below the branch cut. Changing variables to $y=-z-1$, we have

$$
\phi_{n}=\int_{0}^{\infty} \frac{d y}{2 \pi i}(-1)^{n+1} e^{-(n+1) \ln (1+y)} \operatorname{Disc}[\hat{\phi}(-1-y)] .
$$

For $n \gg 1$, the exponential factor cuts off the integral for $y \gg 1 / n$, and so we can use the small $y$ expansion of the rest of the integrand. Then

$$
\begin{aligned}
\hat{\phi}(-1-y \pm i \epsilon) & =\frac{\operatorname{Li}_{1 / 2}(1+y \mp i \epsilon)}{\pi+\mathrm{Li}_{1 / 2}(1+y \mp i \epsilon)} \\
& \approx \frac{\mp i \sqrt{\frac{\pi}{y}}}{\pi \mp i \sqrt{\frac{\pi}{y}}} \\
& \approx \mp i \sqrt{\pi y} .
\end{aligned}
$$

Putting this all together, we get

$$
\phi_{n} \sim \int_{0}^{\infty} \frac{d y}{2 \pi i}(-1)^{n+1} e^{-n y}[\mp 2 i \sqrt{\pi y}]=(-1)^{n} \frac{\Gamma(3 / 2)}{\sqrt{\pi} n^{3 / 2}}=\frac{(-1)^{n}}{2 n^{3 / 2}} .
$$

Hence for the exceptional sampling time $2 \gamma \tau=\pi$ we get $F_{n}=\left|\phi_{n}\right|^{2} \sim n^{-3} / 4$ as reported in the text. As a test, computing $\phi_{20}$ and $\phi_{40}$ numerically, we have $\phi_{20}(20)^{3 / 2}=0.470, \phi_{40}(40)^{3 / 2}=0.484$, which is consistent with a $1 / n$ convergence to $1 / 2$.

\section{The General Calculation}

We now consider the unbounded quantum walk, still starting on the origin, but now for a general sampling rate. Define $\alpha=2 \gamma \tau$ and the function

$$
Q(z, \sigma)=\sqrt{\frac{2}{\pi \alpha}} e^{i \sigma \pi / 4} \operatorname{Li}_{1 / 2}\left(e^{i \sigma \alpha} z\right)
$$

in terms of which we define

$$
R(z)=\frac{1}{2 i}[Q(z, 1)-Q(z,-1)]
$$

We want the coefficient of $z^{n}$ in the Taylor expansion of

$$
\hat{\phi}(z)=\frac{R(z)}{1+R(z)} .
$$

Now, $\hat{\phi}(z)$ has two branch cuts extending along the rays $z_{ \pm}=e^{ \pm i \alpha}(1+y)$ for $y>1$. Note that these coincide in the case $\alpha=\pi$ considered above. $Q(z, 1)$ has a branch cut along $z_{-}$but is finite along $z_{+}$and vice versa. Using our expansion of the polylog, we have

$$
Q\left(z_{+},-1\right) \approx \sqrt{\frac{2}{\pi \alpha}} e^{-i \pi / 4}\left[ \pm i \sqrt{\frac{\pi}{y}}\right]= \pm i \sqrt{\frac{2}{\alpha y}} e^{-i \pi / 4},
$$


and

$$
Q\left(z_{+}, 1\right) \approx \sqrt{\frac{2}{\pi \alpha}} e^{i \sigma \pi / 4} \operatorname{Li}_{1 / 2}\left(e^{2 i \alpha}\right) .
$$

Similarly,

$$
Q\left(z_{-}, 1\right) \approx \sqrt{\frac{2}{\pi \alpha}} e^{i \pi / 4}\left[ \pm i \sqrt{\frac{\pi}{y}}\right]= \pm i \sqrt{\frac{2}{\alpha y}} e^{i \pi / 4}
$$

and

$$
Q\left(z_{-},-1\right) \approx \sqrt{\frac{2}{\pi \alpha}} e^{-i \sigma \pi / 4} \operatorname{Li}_{1 / 2}\left(e^{-2 i \alpha}\right) .
$$

The discontinuity along the $z_{+}$branch cut is then

$$
\operatorname{Disc}\left[\hat{\phi}\left(z_{+}\right)\right] \approx 4 \sqrt{\frac{\alpha y}{2}} e^{i \pi / 4},
$$

and the discontinuity along the $z_{-}$branch is

$$
\operatorname{Disc}\left[\hat{\phi}\left(z_{-}\right)\right] \approx-4 \sqrt{\frac{\alpha y}{2}} e^{-i \pi / 4} .
$$

We thus get

$$
\begin{aligned}
\phi_{n} & \approx \int_{0}^{\infty} \frac{d y}{2 \pi i} e^{-n y}\left[e^{-n i \alpha} \operatorname{Disc}\left[\hat{\phi}\left(z_{+}\right)\right]+e^{n i \alpha} \operatorname{Disc}\left[\hat{\phi}\left(z_{-}\right)\right]\right] \\
& =\frac{1}{2 \pi i n^{3 / 2}}[\Gamma(3 / 2) \sqrt{8 \alpha}] \cdot[-2 i \sin (n \alpha-\pi / 4)] \\
& =-\sqrt{\frac{2 \alpha}{\pi n^{3}}} \sin \left(n \alpha-\frac{\pi}{4}\right) .
\end{aligned}
$$

This immediately gives $F_{n}=\left|\phi_{n}\right|^{2}$ Eq. 17 in the main text. For example, for $\alpha=\pi / 5$, the formula predicts $\phi_{40}(40)^{3 / 2}=\phi_{80}(80)^{3 / 2}=.447$, whereas in truth (obtained with a symbolic program by Taylor expansion of exact result of $\hat{\phi}(z)$ to order $z^{n}$ ) it is 0.458 for $n=40$ and 0.453 for $n=80$, so that the error again falls as $1 / n$. For a non-rational value of $\alpha / \pi$, the formula also works. For example, for $\alpha=1 / 5$, the formula predicts $\phi_{80}=-2.36 \cdot 10^{-4}$, whereas the exact answer is $-2.44 \cdot 10^{-4}$. The strange aspect of the formula is that the limit of $\phi_{n}$ for $\alpha \rightarrow \pi$ does not reproduce the value at $\alpha=\pi$ we found above. This is no doubt due to the presence of two branch cuts for $\alpha \neq \pi$, whereas there is only one at $\alpha=\pi$. This implies that the convergence of the formula for $\alpha$ close to $\pi$ must be a little funny. Thus, for $\alpha=0.95 \pi$, we have that at $n=40$ the ratio of prediction to exact is -2.72 (i.e., even the sign is wrong), whereas for $n=60$ the ratio is 0.705 , for $n=80$, the ratio is 1.03 , at $n=100$, the ratio is 0.947 , and at $n=120$, the ratio is 0.968 , so things are converging, even though even intermediate $n$ 's are way off. The formula also works for $\alpha>\pi$. Thus, for $\alpha=11 \pi / 7$, the ratio is 0.987 at $n=190$. Similar effect takes place for $\alpha$ which is an integer multiple of $\pi$. Finally we note that unlike a classical random walk in one dimension, the eventual probability of being detected is not unity, so even a one-dimensional walk is not recurrent (unless $\tau=0$ which is the trivial case). We will discuss this effect and many others in a longer publication, but for now present $1-S_{\infty}$ versus $\gamma \tau$ in Fig. (4). 


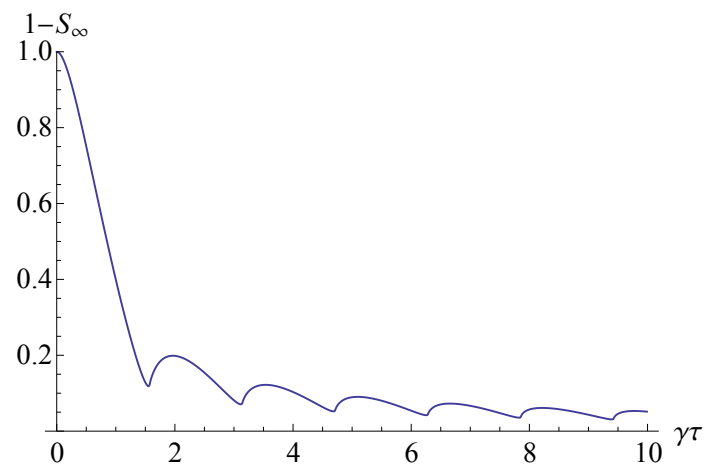

FIG. 4. For the one dimensional tight binding quantum walk the probability that the particle is eventually detected $1-S_{\infty}=$ $\sum_{n=1}^{\infty} F_{n}$ versus the sampling rate $\gamma \tau$ exhibits a non-monotonic behavior. Unlike the classical random walk counterpart the quantum walk is not recurrent, unless $\gamma \tau \rightarrow 0$ which is the trivial case. 